\title{
LARGE AREA SEMI-PERMEABLE ENCAPSULATION MEMBRANES USING CARBON NANOTUBE COMPOSITES
}

\author{
Armon Mahajerin and Liwei Lin \\ Berkeley Sensor and Actuator Center \\ University of California, Berkeley, USA
}

\begin{abstract}
A batch-fabrication methodology to create large area vacuumsealed cavities on top of silicon substrates is reported. This process uses a semi-permeable membrane made of carbon nanotubes (CNTs) and polysilicon. The dense forest of CNTs, conformally coated with polysilicon by low pressure chemical vapor deposition (LPCVD), functions as the semi-permeable membrane. When the top surface is opened via plasma etching of polysilicon, the membrane is penetrable to liquid and vapor. Hydrofluoric acid (HF) vapor is used to etch the underlying oxide before sealing the film with another LPCVD of polysilicon, rendering it impermeable. Sealed areas of more than $1 \times 1 \mathrm{~mm}^{2}$ have been fabricated.
\end{abstract}

\section{INTRODUCTION}

Microelectromechanical systems (MEMS), which function as sensors or actuators, have undergone considerable growth in the past two decades. One of the key developments has been the miniaturization of MEMS, making it possible to integrate their processing with that of integrated circuit (IC) processing to reduce the costs of manufacturing and packaging [1]. However, a key difference between MEMS and IC devices is that MEMS require some form of interaction with their environments for signal processing. This can be problematic when considering process flow compatibility. The key issue for MEMS process development is the packaging of the devices, which is often the primary barrier to the commercialization of MEMS devices. It also tends to contribute to up to $70 \%$ of the total cost for a MEMS device development cycle [2]. Due to the widely varying types of MEMS device materials and applications, a universally adopted industry standard remains elusive. Pressure, chemical, or fluidic sensors should to be open to the environment in order to function. On the other hand, accelerometers, gyroscopes, or oscillators should be hermetically sealed, sometimes with an anti-stiction agent and/or buffer gas [3]. Moisture penetration may also lead to corrosion within the package.

One trend in industry packaging methodologies is the shift from die level packaging to wafer level packaging. Die level encapsulation involves dicing the chips on a wafer then packaging them individually with separate substrates. The drawbacks to this approach include special handling requirements so as to not damage the MEMS devices and reduced throughput since individual dies must first be packaged then tested for meeting performance specifications.

Wafer level packaging mitigates a number of issues in die level packaging [4]. Importantly, incorporating encapsulation into the device fabrication steps prior to dicing provides an inherent protective lid for the MEMS structures during fabrication and back-end assembly. The wafer level encapsulation may be formed by interfacial bonding between the device substrate and a separate sealing wafer or by thin film deposition with the latter approach offering savings in material costs. Thin film encapsulation also eliminates the need for aligning separate wafers as well as the seal ring around each device, thereby increasing available space to enhance yield and throughput. Lastly, the lower topography of the packaged devices permits post encapsulation processing.
The nature of thin films can limiting compared to bonded caps when considering the strength and size of the membrane, since these films may collapse or break under vacuum loads. Some thin film processes use the permeability of that membrane to provide a pathway for etchants to sacrificial layers, and they must be sealed afterward. Other thin films may simply be patterned with a small channel near the base in lieu of permeability [5]. In either case, for larger areas it's often necessary to bolster the membrane with a hard film like silicon nitride, which may be problematic for processing due to slow deposition.

Polysilicon is one such material that can be made permeable for thicknesses around less than $1 \mu \mathrm{m}$ [6,7]. However, incorporating this into a fabrication process requires numerous steps of doping, annealing, hard mask under layers, etc. that can be tedious. Thickness and area limitations are also an issue. However, combining polysilicon with another material such as CNTs opens possibilities to use thicker films for larger areas. The results presented here aim to improve the flexibility in designing the thickness and strength of encapsulation membranes compared to other thin film approaches. The concept of using a composite film of polysilicon and CNTs is discussed in the following section.

\section{CONCEPT}

CNTs are attractive materials for a number of reasons. They may be grown with very large aspect ratios and possess semiconducting properties along with good mechanical strength and self-alignment when grown in a forest. In this paper's process CNT height may be set anywhere from $5 \mu \mathrm{m}$ to $50 \mu \mathrm{m}$ with short processing times, with CNTs serving as a skeletal framework for deposited encapsulation films. Moreover, the inclusion of CNTs into a composite film generates a natural porosity in the film due to the spacing between individual CNTs. Thus, a semi-permeable membrane forms by coating the CNT forest with a material like polysilicon. Fig. 1 below shows a schematic of the composite film.

\section{CNT/Polysilicon Encapsulation Membrane}

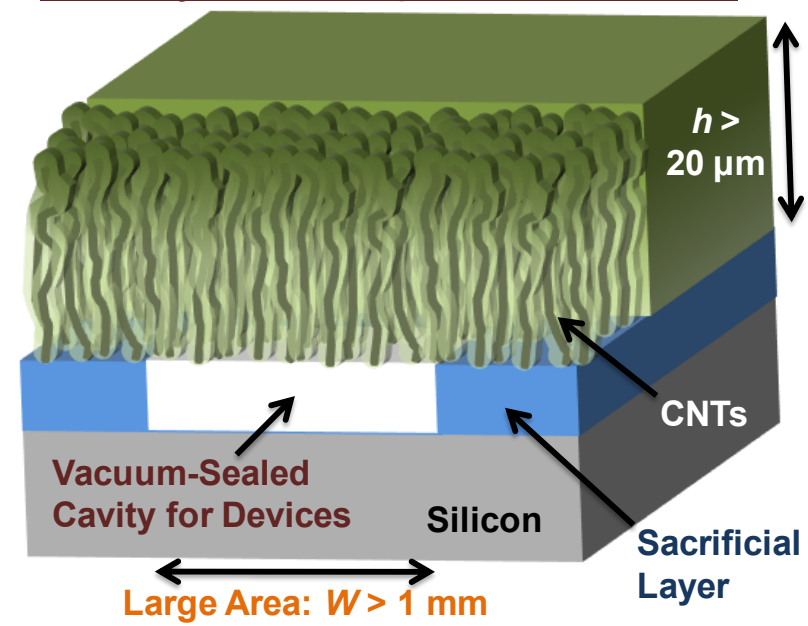

Figure 1: Conceptual illustration of encapsulation using a semipermeable CNT/polysilicon membrane. 


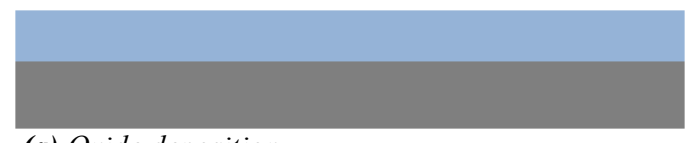

(a) Oxide deposition

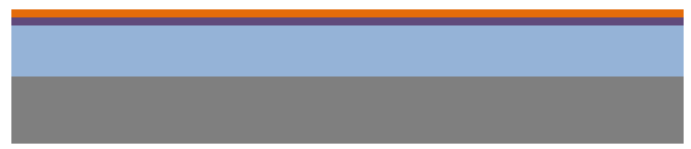

(b) Evaporation of Al and Fe films

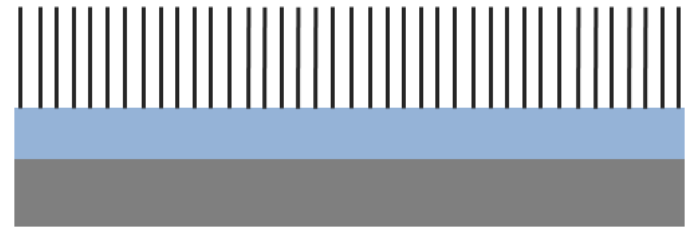

(c) CNT growth

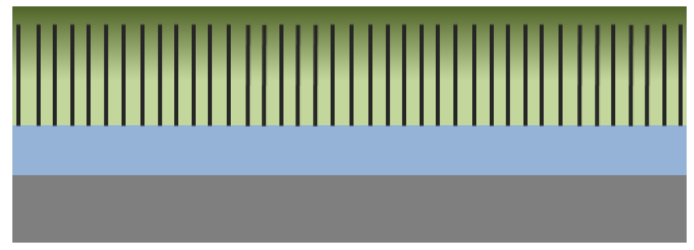

(d) First polysilicon LPCVD

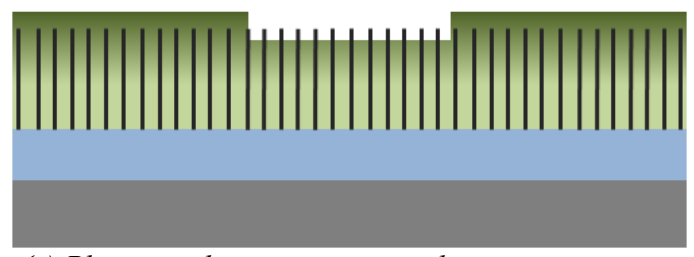

(e) Plasma etching to create window

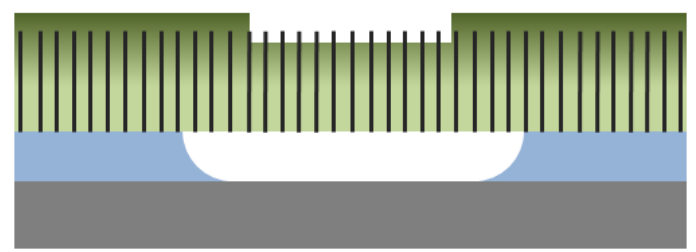

(f) HF vapor etching of oxide

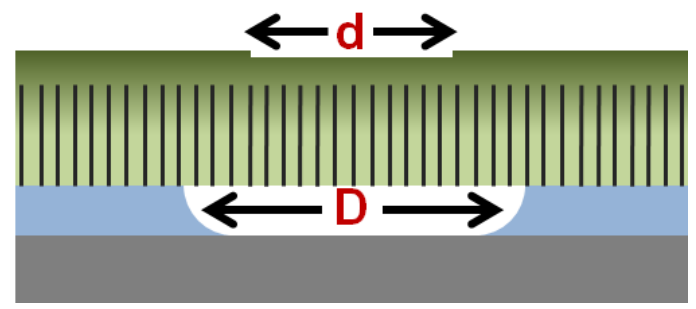

(g) Second polysilicon LPCVD (sealing)

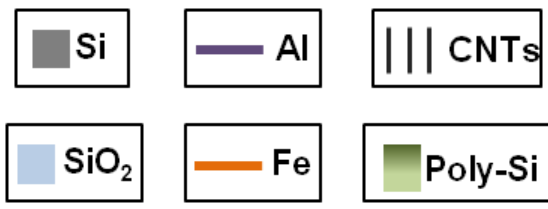

Figure 2: Fabrication process for developing the CNT/polysilicon composite and forming the sealed cavity.

\section{FABRICATION}

The process flow for testing the $\mathrm{CNT} /$ polysilicon composite, performing oxide etching, and sealing the cavity is shown in Fig. 2. The fabrication begins with a bare silicon wafer. Low-temperature oxide is deposited as a sacrificial layer onto the substrate via LPCVD in a furnace at a process temperature of $450^{\circ} \mathrm{C}$. The deposition rate is roughly $700 \mathrm{~nm} /$ hour. In a fully realized MEMS process the device should be embedded within the oxide. If much thicker films are needed, oxide from a liquid source of tetraethyl orthosilicate (TEOS) may be deposited with plasma enhanced chemical vapor deposition (PECVD) to drastically reduce the deposition time compared to a furnace process.

Next, two metal films are deposited using electron-beam evaporation at a pressure of $10^{-6}$ torr or less (Fig. 2b). First, an aluminum film of $10 \mathrm{~nm}$ is evaporated, followed by $5 \mathrm{~nm}$ of iron. The Al layer serves as a barrier against interaction between the iron and the substrate underneath. In addition, Al also supports proper nucleation of $\mathrm{Fe}$, which serves as the catalyst for the CNT growth process.

After metal deposition the CNTs may be grown in a simple furnace at a temperature of $720^{\circ} \mathrm{C}$ with hydrogen $\left(\mathrm{H}_{2}\right)$ and ethylene $\left(\mathrm{C}_{2} \mathrm{H}_{4}\right)$ gas flow (Fig. 2c). After approximately two minutes CNTs begin to grow at a rate of roughly $5 \mu \mathrm{m} / \mathrm{min}$. One should note that this growth rate is sensitive to the thickness of the iron film, but a $30 \mu \mathrm{m}$ CNT height may typically be grown in less than $8 \mathrm{~min}$. Fig. 3 shows two different heights for CNT forests, both of which are vertically aligned and dense. CNT forests as tall as $50 \mu \mathrm{m}$ have been successfully grown as well. Depending on the required area for a particular application, modulating the CNT height is quite easy, compared to various thin film approaches which may require different materials or steps in order to thicken the film and make it more robust.

Once the CNT forest is in place, the first LPCVD of polysilicon occurs with a deposition temperature is $615^{\circ} \mathrm{C}$ (Fig. $2 \mathrm{~d}$ ). The minimum time for this step was found to be $45 \mathrm{~min}$ in order to fill the top portion of the CNT forest fully with polysilicon. Fig. 4 shows the coating behavior of the polysilicon throughout the height of the CNTs. The thickness of deposited polysilicon is lower near the bottom of the forest primarily because the top $5 \mu \mathrm{m}$ fill more quickly, thereby constricting gas diffusion through the forest. This is favorable in that the polysilicon forms a natural seal at the top of the composite film.
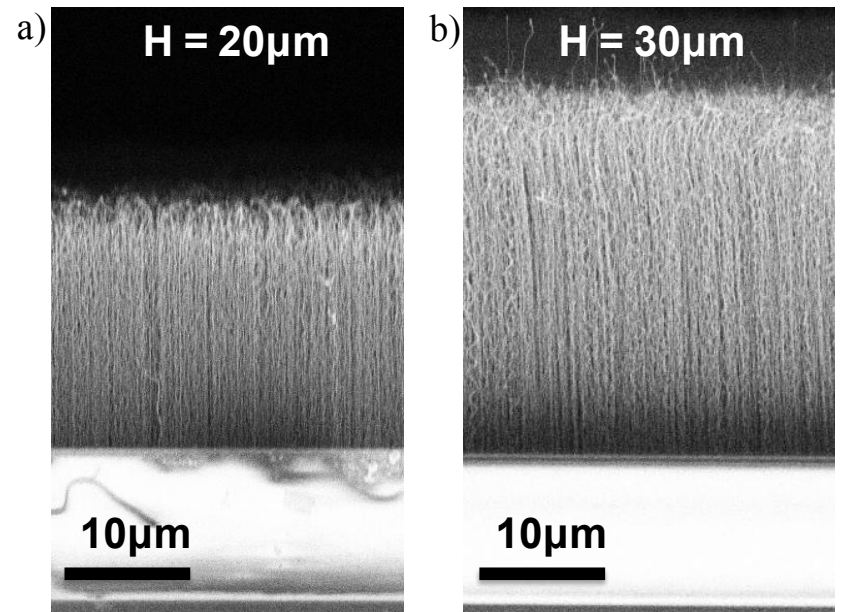

\section{$10 \mu \mathrm{m}$}

Figure 3: SEM photographs showing CNT forest (a) after 4 min growth, resulting in a $20 \mu \mathrm{m}$ tall forest; and (b) after 5 min growth, resulting in a $30 \mu \mathrm{m}$ tall forest. 


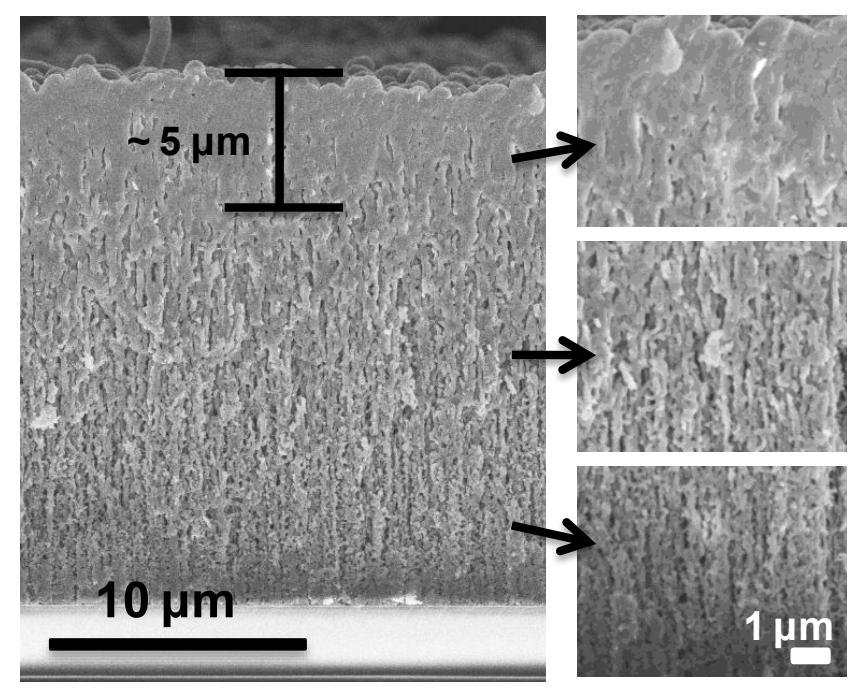

Figure 4: SEM photograph showing varying thickness of deposited polysilicon on CNTs from top to bottom as well as naturally occurring pores that result from the polysilicon coating.

Next, a select area on top of the composite must be etched to remove the polysilicon (Fig. 2e). This opens the vertical pathway for liquids and gas to travel through the membrane to the underlying material. A gas mixture of $90 \%$ sulfur hexafluoride $\left(\mathrm{SF}_{6}\right)$ and $10 \%$ oxygen $\left(\mathrm{O}_{2}\right)$ flowing at $66 \mathrm{sccm}$ will etch polysilicon at roughly $0.45 \mu \mathrm{m} / \mathrm{min}$ under $50 \mathrm{~W}$ power. The top surface of the film darkens as polysilicon is removed, indicating the CNTs have been exposed. The profile of the opened etchant access window may be seen in Fig. 5. Rectangular cavity windows as wide as $1250 \mu \mathrm{m}$ along the shorter direction were used.

Once the pores in the composite film are exposed, sacrificial oxide is removed with HF vapor penetration (Fig. 2f). The chip or wafer is set onto an electrostatic chuck, held at $45^{\circ} \mathrm{C}$, and placed over the $\mathrm{HF}$ vapor bath, which is kept at $35^{\circ} \mathrm{C}$, in order to prevent condensation of HF or reaction byproducts within the cavity. Lastly, another polysilicon LPCVD of $45 \mathrm{~min}$ seals the film and the newly created cavity (Fig. 2g).

\section{EXPERIMENTAL RESULTS}

\section{HF Vapor Etching of Underlying Oxide}

HF vapor penetration occurred almost instantly during experiments and cleared out buried oxide at a rate of nearly 1 $\mu \mathrm{m} / \mathrm{min}$. Cavities wider than $1 \mathrm{~mm}$ may be generated in less than $6 \mathrm{~min}$. However, there is a trade-off between the thicknesses of the oxide layer and the composite film. Specifically, as the oxide thickness increases to $10 \mu \mathrm{m}$ and beyond, lateral undercut or overetch occurs. Over-etch is defined based on the width of the window, $d$, and the width of the resultant cavity, $D$ as indicated in Fig. 2g. Then,

$$
\% O E=\frac{D-d}{d}
$$

Two sets of composite films with differing CNT heights of 20 $\mu \mathrm{m}$ tall and $30 \mu \mathrm{m}$ were tested against over-etching. The thinner membrane exhibited far less over-etch, up to $12 \%$, as a function of increasing exposure to $\mathrm{HF}$ vapor. On the other hand, the thicker membrane reached over-etch values of almost $100 \%$, or twice the intended cavity width as defined by the window on the composite membrane. The primary reason for this is that the permeability of the $\mathrm{CNT}$ /polysilicon composite comes from the pores in the

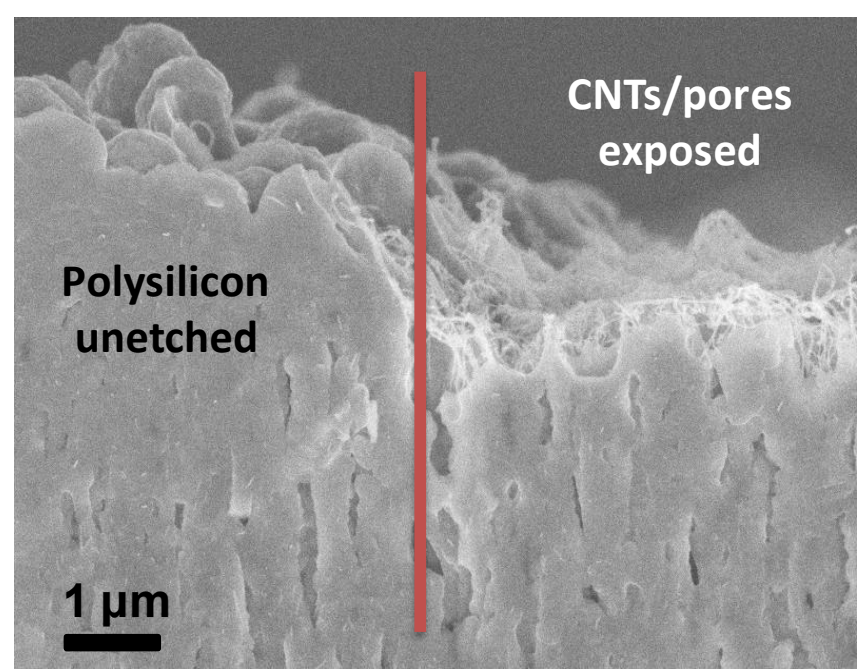

Figure 5: SEM photograph of composite film's top surface profile after plasma etching, which exposes CNTs and opens the underlying pores to liquid and vapor.

membrane and not the CNTs themselves. Therefore, there is a degree of lateral permeability in the membrane, evident even when HF vapor time is minimal ( $2 \mathrm{~min})$. The extent of over-etch may be modulated by reducing the thickness, essentially the CNT forest height. However, reducing thickness also reduces strength and limits how large an area may be safely sealed without membrane collapse. Nevertheless, the fabrication of the composite film is versatile enough such that changing the CNT height for different applications is seamless.

\section{Sealing the Membrane}

The second polysilicon only deposited onto the top of the membrane, as the top section is nearly filled from the first polysilicon deposition even after plasma etching. This is important for ensuring that polysilicon does not deposit onto the surface of the cleared cavity substrate or a device contained within. In addition, it's noted that defining the etch window creates a step height of roughly $2-3 \mu \mathrm{m}$ on top of the composite membrane. This is not an issue for the sealing step after oxide removal, but for subsequent post-encapsulation processing a planar surface may be required. In this case the top surface of the membrane may be smoothened with self-planarizing TEOS deposition step. This permits post-seal processing steps such as opening electrical contacts.

A surface profilometer was used to examine the membranes for possible deflection after sealing with LPCVD due to the pressure difference between inside the cavity and outside atmosphere. This deflection is a function of the membrane thickness and cavity width. For example, in the case of $1000 \mu \mathrm{m}$ wide etch windows, the membrane built with $25 \mu \mathrm{m}$ tall CNTs showed a concave up membrane shape with maximum deflection of $7 \mu \mathrm{m}$ in the center of the cavity. Increasing the CNT height to $45 \mu \mathrm{m}$ for the same cavity size improved the strength of the film against pressure loading with no measurable curvature of deflection. These results take into account the step height between the window and unetched areas as discussed above.

\section{CONCLUSIONS}

Wafer level encapsulation using a CNT/polysilicon composite film improves upon the flexibility in designing the thickness and area of an encapsulation membrane compared to other thin film 
approaches. The membrane contains a natural porosity that facilities removal of sacrificial oxide via $\mathrm{HF}$ vapor. The fabrication of the CNT/polysilicon composite is highly tunable with regard to the CNT forest height that supports the membrane. Etching behavior of buried oxide using HF vapor was characterized for different membrane thicknesses, based on the CNT heights, confirming that lateral over-etch may be reduced by using shorter CNTs. For large area applications (e.g. $>1 \mathrm{~mm}^{2}$ ) in which thicker CNTs are needed to withstand against deflection, the etch window may be designed smaller than the intended cavity size to compensate for lateral undercut. Overall the process technology shows promise and versatility for a simplified approach to waferlevel encapsulation of MEMS devices.

\section{ACKNOWLEDGEMENTS}

Travel support has been generously provided by the Transducer Research Foundation.

\section{REFERENCES}

[1] R.N. Candler, W.-T. Park, H. Li, G. Yama, A. Partridge, M. Lutz, and T.W. Kenny, "Single Wafer Encapsulation of MEMS Devices", IEEE Transactions on Advanced Packaging, 26, 3 (2003).
[2] H.M. Clearfield, J.L. Young, S.D. Wijeyesekera, and E.A. Logan, "Wafer-Level Chip Scale Packaging: Benefits for Integrated Passive Devices", IEEE Transactions on Advanced Packaging, 23, 2 (2000).

[3] S.-H. Choa, "Reliability of MEMS packaging: vacuum maintenance and packaging induced stress", Microsystem Technologies, 11 (2005).

[4] M. Esashi, "Wafer level packaging of MEMS", Journal of Micromechanics and Microengineering, 18 (2008).

[5] C. O'Mahony, M. Hill, Z. Olszewski, and A. Blake, "Waferlevel thin-film encapsulation for MEMS", Microelectronic Engineering, 86 (2000).

[6] R. He and C.-J. Kim, "On-Wafer Monolithic Encapsulation by Surface Micromachining With Porous Polysilicon Shell", Journal of Microelectromechanical Systems, 16, 2 (2007).

[7] K.S. Lebouitz, A. Mazaheri, R.T. Howe, and A.P. Pisano, "Vacuum Encapsulation of Resonant Devices Using Permeable Polysilicon", Technical Digest of the $12^{\text {th }}$ Annual IEEE International Conference on MEMS (1999), pp. 470475 .

\section{CONTACT}

*A. Mahajerin, tel: 1-510-642-8983; armonmah@me.berkeley.edu 